Case Report

OOpen Access

CrossMark

\title{
A fatal complication of colonoscopy due to a stenotic colon cancer: an alert to colleagues
}

\begin{abstract}
Colonoscopy is the most commonly performed method for Colon Cancer diagnosis and screening. And a successful exam requires bowel preparation. One of the main contraindications of the use of bowel cleansing agents is intestinal mechanical obstruction. But in many scenarios, as in public health services, there is a delay between the medical exam request and the data of the procedure. In some countries, this delay could be of many months. This interval is enough for nonobstructive colon cancer to turn into an obstructive lesion. The intake of bowel cleansing agents in an impending obstructive bowel syndrome could be a catastrophe. This is illustrated by this case report.
\end{abstract}

Volume 12 Issue 5 - 202I

\author{
Pedro Grachinski Buiar,' Marcela de Rezende \\ Karnikowski \\ 'Pharmaceutic Sciences Post Graduation, State University of \\ Ponta Grossa, Brazil \\ ${ }^{2}$ Emergency Department, Hospital São Lucas da PUCRS \\ (Pontifícia Universidade Católica do Rio Grande do Sul), Brazil
}

\begin{abstract}
Correspondence: Pedro Grachinski Buiar, MD, MSc; Pharmaceutic Sciences Post Graduation Department, State University of Ponta Grossa, Avenida Carlos Cavalcanti, 4748, Uvaranas - Zip code 84030-900, Tel +55 42 3026-0203, Email pgbuiar@gmail.com
\end{abstract}

Received: September 21, 202I | Published: October 22, 202 I

\section{Introduction}

According to World Health Organization, Colorectal Cancer is the third most diagnosed cancer in males and the second in females around the world. Despite being discovered commonly after 55 years, the incidence among young adults is increasing. ${ }^{1}$ Among the risk factors for colorectal cancer, we have obesity, read, and processed meat consumption. ${ }^{2,3}$ The standard diagnostic procedure for colon cancer is colonoscopy, a safe procedure under normal conditions but that has some important contraindications. One of them being the suspicion of bowel obstruction.

\section{Case presentation}

A 74-year-old hypertensive female, with no other comorbidities, was brought to emergency due to intense pain in her legs and massive abdominal distension. Her last bowel movement was four days ago, with liquid feces in a small amount. Familiars referred bowel habit alternation 2 months before associated to $10 \mathrm{Kg}$ weight loss. They also informed that the night before she initiated an oral bowel cleansing agent for an outpatient diagnostic colonoscopy requested one month ago by the primary care physician. When she presented to the emergency department, the patient was hemodynamically unstable, hypotensive, with conscience alteration, presenting fecaloid vomiting and a massively distended abdomen. Rapid laboratory tests revealed a serum lactate level of $15.9 \mathrm{mmol} / \mathrm{L}$ (reference range $0.4-2.0$ $\mathrm{mmol} / \mathrm{L}$ ). An emergency abdominal CT scan was ordered (Figure 1).

CT scan revealed severe dilatation of the small bowel and colon (reaching $15 \mathrm{~cm}$ in greatest diameter) with a stenotic colonic lesion in splenic angle (Figure 2). Despite fluid resuscitation and clinical measures to relieve the intra-abdominal pressure, the patient rapidly evolved with cardiopulmonary arrest and death.
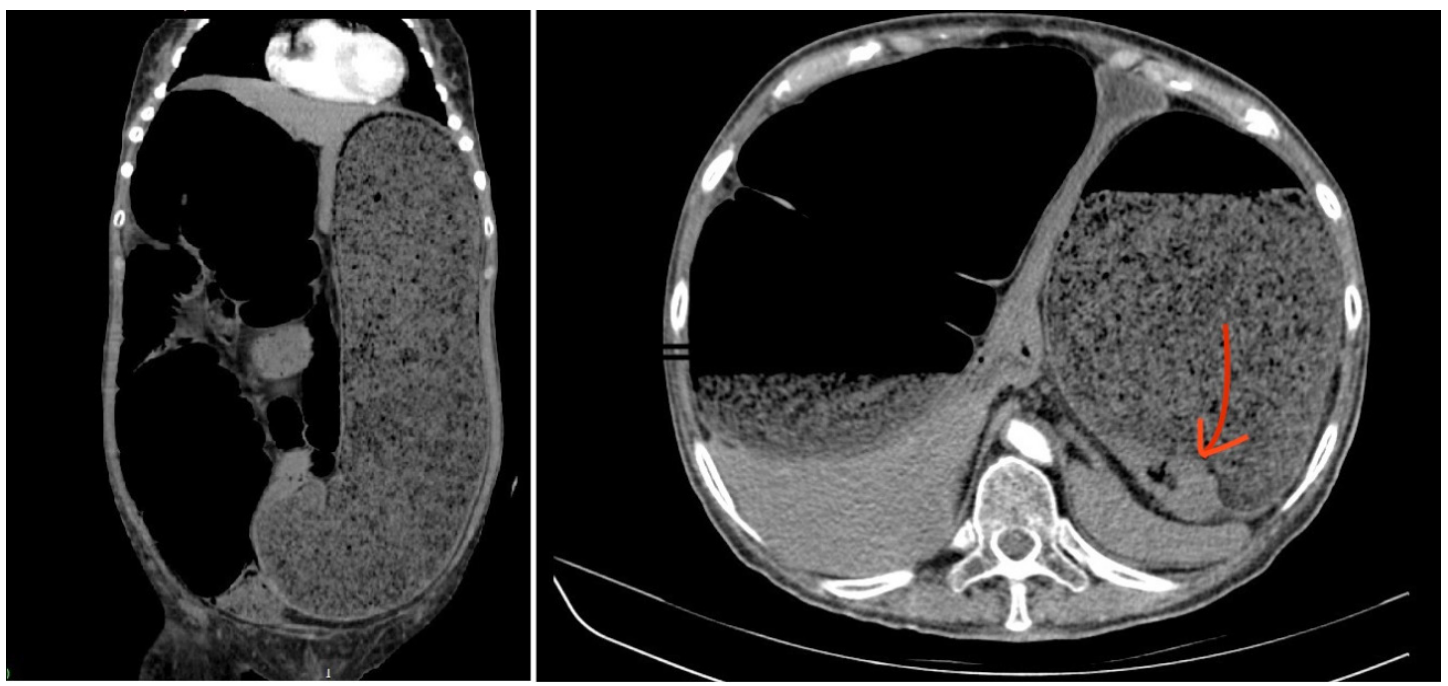

Figure I (A) Massive Intestinal Distension and (B) reduced lung capacity. 


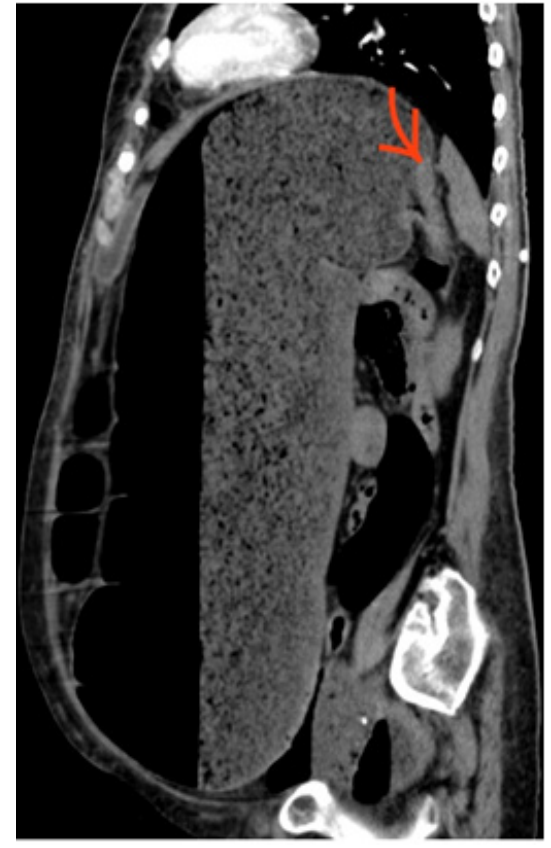

Figure 2 Presence of a stenotic tumoral lesion in the left colon.

\section{Discussion}

Large bowel obstruction is a medical emergency potentially fatal if not correctly or promptly diagnosed, and depending on aggravating factors like that described in this report. The main causes of large bowel obstruction include diverticulitis, volvulus, and colorectal cancer, with malignancy being responsible for the majority of cases. ${ }^{4}$

At least $7 \%$ of all colorectal cancers present initially with an obstructive syndrome..$^{5}$ In these cases, the mortality rate could reach $10 \%$, and the standard emergency colectomy treatment within 24 hours has an associated mortality rate near $22 \%{ }^{6}$

In the earlier stages, colorectal cancer can present with symptoms less noticed by the patient, like weight loss (18\%) and bowel habits changing (58\%). The commonly cited abdominal pain is present in only $24 \%$ in the early stages. ${ }^{7}$ The bowel habits alternation is the symptom that is most associated with a delay in the diagnosis. And among the small group of patients that report this symptom, only $12.4 \%$ attributed this symptom to Cancer. ${ }^{8}$

The standard method to visualize the intestinal mucosa and confirm the diagnosis of colon cancer is the colonoscopy. Bowell preparation regimens involve changes in dietary patterns and the use of oral cathartics. Those can be hypoosmotic, isosmotic or hyperosmotic. Gastrointestinal obstruction is a definitive contraindication to the administration of oral bowel-cleansing agents. ${ }^{9}$ Many medical centers face difficulties with the agenda of colonoscopy. And is very common long waiting times, more than would be tolerable. ${ }^{10,11}$

This patient was waiting for her colonoscopy with symptoms of intestinal partial occlusion. And then she became completely obstructed, without a suspicion. The patient started the bowel cleansing agent prescribed weeks before in the scheduled time and this combination led to a compartment syndrome due to the accumulation of intestinal content in the presence of an obstruction of the intestinal transit.

This case illustrates a lethal association of impending malignant bowel obstruction with the use of osmotic laxatives. A combination of factors that led to this catastrophic outcome. Therefore, we must always bear in mind the contraindications of bowel cleansing agents, especially in crowded health systems that are saturated and with long waiting times.

\section{Acknowledgments}

None.

\section{Conflicts of interest}

Authors declare that there is no conflict of interest.

\section{References}

1. Siegel RL, Fedewa SA, Anderson WF, et al. Colorectal Cancer Incidence Patterns in the United States, 1974-2013. J Natl Cancer Inst. 2017;109(8):djw322.

2. Karahalios A, English DR, Simpson JA. Weight change and risk of colorectal cancer: a systematic review and meta-analysis. Am J Epidemiol. 2015;181(11):832-845.

3. Bouvard V, Loomis D, Guyton KZ, et al. Carcinogenicity of consumption of red and processed meat. International Agency for Research on Cancer Monograph Working Group. Lancet Oncol. 2015;16(16):1599-1600.

4. Yeo HL, Lee SW. Colorectal emergencies: Review and controversies in the management of large bowel obstruction. $J$ Gastrointest Surg. 2013;17(11):2007-2012.

5. Dauphine CE, Tan P, Beart RW Jr, et al. Placement of self-expanding metal stents for acute malignant large-bowel obstruction: a collective review. Ann Surg Oncol. 2002;9(6):574-579.

6. Barillari P, Aurello P, De Angelis R, et al. Management and survival of patinets with obstructive colorectal cancer. Int Surg. 1992;77(4):251-255.

7. Smith D, Ballal M, Hodder R, et al. Symptomatic presentation of early colorectal cancer. Ann R Coll Surg Engl. 2006;88(2):185-190.

8. Mor V, Masterson-Allen S, Goldberg R, et al. Pre-diagnostic symptom recognition and help seeking among cancer patients. Journal of Community Health. 1990;15(4):253-266.

9. Connor A, Tolan D, Hughes S, et al. Consensus guidelines for the safe prescription and administration of oral bowel-cleansing agents. Gut. 2012;61(11):1525-1532.

10. Viiala CH, Tang KW, Lawrance IC, et al. Waiting times for colonoscopy and colorectal cancer diagnosis. Med J Aust. 2007;186(6):282-285.

11. Sey MS, Gregor J, Adams P, et al. Wait times for diagnostic colonoscopy among outpatients with colorectal cancer: a comparison with Canadian association of gastroenterology targets. Can $J$ Gastroenterol. 2012;26(12):894-896. 